\title{
Communication satellite resource scheduling based on improved whale optimization algorithm
}

\author{
Wenwen Liu*, Wei Xiong, and Chi Han \\ Space Engineering University Science and Technology on Complex Electronic System Simulation \\ Laboratory, Beijing, China
}

\begin{abstract}
Under the background of increasing pressure of satellite communication support, reasonable and efficient dispatch of communication satellite resources is an important means to improve the utilization efficiency of communication resources. Aiming at the resource scheduling task requirements of geostationary orbit communication satellite system, communication satellite resource scheduling (CSRS) model is established first of all, based on this, advances a kind of CSRS method based on improved whale optimization algorithm. In this method, the detection and search strategy is proposed and the crossover mutation operator is used to avoid the algorithm falling into local optimum. Simulation results show that IWOA can effectively improve the quality and stability of satellite resource scheduling.
\end{abstract}

Keywords: Communication satellite, Resource scheduling, Improve whale optimization algorithm.

\section{Introduction}

Satellite communication refers to the communication method that uses satellites as relay stations to relay radio signals between multiple earth stations or user terminals, thus realizing information exchange and information transmission between them. After entering the 21 st century, GEO broadband satellite communication system develops rapidly and provides a flexible and efficient satellite communication mode for the interconnection application of high data bandwidth and multimedia. It is featured by wide coverage, high bandwidth, flexible networking mode and high reliability, and is the frontier technology and development hotspot in the application field of satellite communication.

Whether military or civilian, satellite communication is in urgent demand. However, the current broadband communication satellite system in China is in the construction stage, and the communication capacity is insufficient, so it is necessary to meet more task requirements as far as possible under the circumstance of limited resources. In recent years, intelligent optimization algorithms have been used to solve multi-satellite and multi-task problems. Li Hongjun et al.[1] adopted tabu search algorithm to solve the multi-star and

${ }^{*}$ Corresponding author: 19910398967@163.com 
multi-task problem. He Yin et al.[2]introduced ant colony algorithm into the problem of satellite communication resources and task matching, which improved the comprehensive income of task scheduling. Lin Yusheng et al.[3]used genetic algorithm to solve the multitask single satellite resource scheduling problem.

The above algorithms can solve the communication satellite resource scheduling problem well, but with the increasing complexity of communication tasks and satellite resources, the performance requirements of optimization algorithms are becoming higher and higher. Researchers continue to study new algorithms and improve existing algorithms to better adapt to the development of scheduling problems. In 2016, Mirjalili et al.[4]proposed Whale Optimization Algorithm by imitating the humpback Whale bubble net hunting strategy. WOA). At present, this algorithm has been applied to image segmentation[5], location optimization[6], fault diagnosis[7] and achieved good results. In this paper, whale algorithm is applied to solve the communication satellite resource scheduling problem to obtain the high quality resource scheduling scheme.

\section{Analysis of communication satellite resource scheduling model}

\subsection{Resource scheduling scenario analysis}

Communication satellite resources refer to the frequency resources within a certain time range. The rectangle in Figure 1 represents the task, the length of the rectangle represents the length of the task time, the width represents the bandwidth of the task, and the X-axis coordinate in the lower left corner of the rectangle represents the start time of the task. When tasks apply for requirements, they will overlap in time or frequency, resulting in resource conflicts. Satellite resource scheduling is to resolve conflicts on the premise of meeting task requirements as much as possible, achieve the goal of communication resource allocation, and finally make communication resource allocation orderly, as shown in Figure 2.

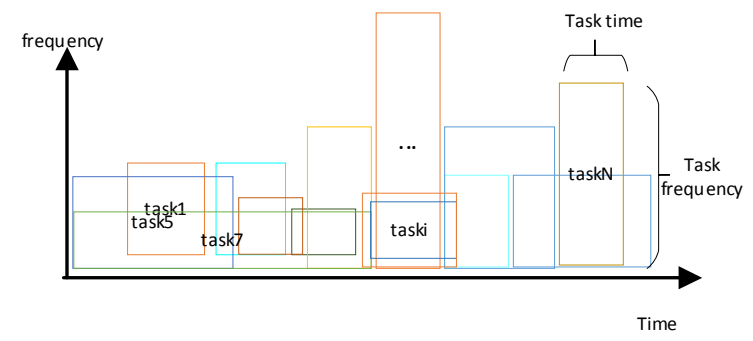

Fig. 1. Schematic diagram of task requirement conflict.

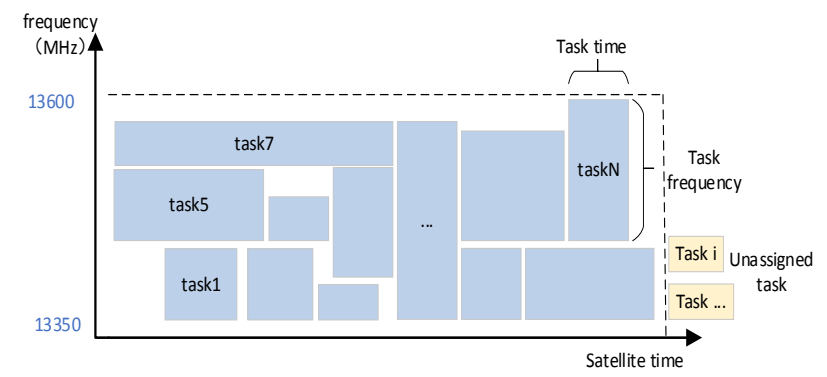

Fig. 2. Schematic diagram of allocation results of communication satellite resource. 


\subsection{Parameter definition}

Communication satellite resource scheduling is a complex combinatorial optimization problem. In order to facilitate the elaboration of the model, the definitions of parameters in the scheduling problem are given below:

\subsubsection{Definition of task information}

In the process of resource allocation, the task model includes frequency band, frequency band bandwidth, task start time, task end time, task duration and task area, $T$ is task set and $N$ is the number of tasks. The task $i$ is denoted as $T_{\mathrm{i}}=\left\{T_{D i}, t b_{i}, t e_{i}, t_{i}, T F_{i}, b_{i}, p_{i}\right\}$, $1 \leq \mathrm{i} \leq N$, and the specific explanation is shown in Table 1 .

Table 1. Symbol description of main parameters of task requirements.

\begin{tabular}{cccc}
\hline Symbol & Paraphrase & Symbol & Paraphrase \\
\hline$T$ & Task set & $T_{\mathrm{i}}$ & Task i \\
$T_{D \mathrm{i}}$ & The task area of task i can be represented & $t_{i}$ & Duration of task i \\
& by latitude and longitude & $t e_{i}$ & End time of task i \\
$\mathrm{tb}_{\mathrm{i}}$ & The start time of task i & $b_{i}$ & Bandwidth of task i \\
$T F_{i}$ & Task i operating frequency band & $N$ & Number of tasks \\
$p_{i}$ & Task i Task priority & & \\
\hline
\end{tabular}

\subsubsection{Definition of resource information}

The single repeater resource model can be represented by a multidimensional variable, The satellite is represented by $S=\{F, f b, f e, B, T b, T e, D\}$. Table 2 describes the parameters.

Table 2. Symbol description of main parameters of task requirements.

\begin{tabular}{|c|c|c|c|}
\hline Symbol & Paraphrase & Symbol & Paraphrase \\
\hline$S$ & Repeater resource & $F$ & The frequency band of the repeater \\
\hline$f b$ & $\begin{array}{l}\text { Start frequency of } \\
\text { the repeater }\end{array}$ & $f e$ & Termination frequency of the repeater \\
\hline$B$ & $\begin{array}{l}\text { Bandwidth of the } \\
\text { forwarder }\end{array}$ & $T b$ & Start time of forwarder availability \\
\hline$T e$ & $\begin{array}{l}\text { Available end time } \\
\text { of the forwarder }\end{array}$ & $D$ & $\begin{array}{c}\text { The coverage area of the repeater can be } \\
\text { expressed by latitude and longitude }\end{array}$ \\
\hline
\end{tabular}

\subsubsection{Variable definition}

Definition $X$ is the set of decision variables for all tasks, $X=\left\{x_{1}, x_{2}, x_{3} \cdots x_{N}\right\}, x_{i}=1$ indicates that the task $i$ is allocated to resources and can be executed; $x_{i}=0$ indicates that the task $i$ cannot be executed because no resources are allocated to it. 


\subsection{Resource scheduling model construction}

\subsubsection{Scheduling target}

Maximum total benefit of task scheduling:

$$
C_{r}=\max \sum_{i=1}^{N} x_{i} p_{i}^{-1}
$$

Maximum number of tasks performed:

$$
C_{l}=\max \sum_{i=1}^{N} x_{i}
$$

where, $N$ is the number of tasks, $x_{i}$ is the decision variable of the task $i$, and $p_{i}^{-1}$ is the reciprocal of the priority of the task, which is used to represent the profit value of the task.

In this paper, the two targets are added together according to the weight to form a comprehensive single target:

$$
C=\alpha \max \sum_{i=1}^{N} x_{i} p_{i}^{-1}+\beta \max \sum_{i=1}^{N} x_{i}
$$

where, $\alpha, \beta$ is the weight of the objective function, $\alpha=\beta=0.5$.

\subsubsection{Mathematical model of communication satellite resource scheduling}

Communication satellite resource scheduling can be modeled as the following mathematical model:

$$
\begin{gathered}
C=\alpha \max \sum_{i=1}^{N} x_{i} p_{i}^{-1}+\beta \max \sum_{i=1}^{N} x_{i} \\
\text { s.t. } T_{D i} \in D \\
T_{b} \leq t b_{i}, t e_{i} \leq T_{e} \\
T F_{i} \in F \\
b_{i} \leq B
\end{gathered}
$$

The following is a description of the model: Constraint (9) describes that the task area must be within the repeater coverage. Constraint (10) The execution time used to describe the task must be within the forwarder available time range. Restriction (11) The frequency band required by the task must be the same as the repeater frequency band. Constraint (12) Indicates that the amount required by the task must be less than or equal to the bandwidth of the forwarder. 


\section{Improving the whale optimization algorithm}

The Whale Optimization Algorithm (WOA) is an algorithm based on the behavior of whales that round up their prey. In WOA, the position of each whale represents a feasible solution. During a group hunt, each whale does two things. One is to surround the prey, and all the whales move toward the other whales. The other is a bubble net, in which the whale swims in a circular circle to expel air bubbles from its prey. In each generation of swims, whales randomly choose these two behaviors to hunt [8]. Whale algorithm has the advantages of few control parameters, simple structure, easy implementation and convenient calculation.

\subsection{Coding}

The number of tasks is $N$. After tasks are sorted according to certain rules, the number of tasks is $1,2,3,4 \cdots N$, and $T_{\mathrm{i}}$ represents task $\mathrm{i}$. The number of satellite resources is $M$. After the satellite resources are sorted according to certain rules, the number of satellite resources is $S_{1} S_{2} \cdots S_{j} \cdots S_{M} \cdot S_{\mathrm{j}}$ represents satellite resources $j$. After coding, select satellite resources for the task, and finally form the coding sequence $S=\left\{S_{3}, S_{5}, S_{3}, S_{\mathrm{M}} \cdots S_{4}\right\}$ of satellite resource allocation according to the task sequence.

\subsection{Initial solution generation}

According to the method of random sequence generation, the population $P_{1}$ with population quantity $N_{P}$ is generated, and the individuals in $P_{1}$ are reversed to form population $P_{2}$. The $P_{1}$ and $P_{2}$ are combined into population $P_{3}$, Then, the fitness function of the remaining individuals is calculated, and the individuals with higher fitness function are selected to form the final initial population $P$.

\subsection{Adaptive degree value}

For a given whale location, the fitness function is defined as:

$$
F=\alpha C_{r}+\beta C_{l}
$$

\subsection{Encirclement search}

Whales need to gather when surrounding their prey, and each whale has two choices of movement direction[9] :

One is to approach the best individual in the population. Select the individual $X_{\text {best }}$ with the optimal position in the current population, and other individuals $X(t)$ can choose to approach the optimal individual to improve the quality of individual position. The selection method is expressed by the following formula:

$$
\begin{aligned}
& D=\left|C \cdot X_{\text {best }}(t)-X\right| \\
& X(t+1)=X_{\text {best }}-A \cdot D
\end{aligned}
$$


where, $t$ is the number of iterations, and $A, C$ is the coefficient matrix, and the expression is:

$$
\begin{aligned}
& A=2 a \cdot r_{1}-a \\
& C=2 * r_{2} \\
& a=2-t * 2 / \mathrm{t}_{\max }
\end{aligned}
$$

where, $r_{1}, r_{2}$ is a random number from 0 to $1 . t_{\max }$ is the maximum iteration number of the algorithm.

The other is to randomly choose the direction to swim. The current individual $X(t)$ randomly selects $X_{\mathrm{abs}}(t)$ body to approach. The formula of this selection mode is expressed as:

$$
\begin{aligned}
& D=\left|C \cdot X_{a b s}(t)-X\right| \\
& X(t+1)=X_{a b s}-A \cdot D
\end{aligned}
$$

\subsection{Bubble net drive}

When whales hunt, they will eject bubbles to form bubble nets to drive away prey[10]. When using bubble nets to drive prey, whales will constantly update their position. When using bubble nets, the whale's position is updated by the following formula:

$$
\begin{gathered}
X(t+1)=\left|X_{\text {best }}-X(t)\right| \cdot e^{b l} \cdot \cos (2 \pi l)+X_{\text {best }} \\
l=\left(-1+t *-1 / t_{\max }\right) * r_{3}+1
\end{gathered}
$$

where, $b$ is used to define the shape of spiral, which is $1 . r_{3}$ is a random number from 0 to 1.

\subsection{Reconnaissance search}

To whales algorithm two kinds of search mode analysis: surrounded by search near the optimal individual and bubble net are all around the best individual search, search the random choice of the individual is surrounded around random individual search, whale algorithm are major search around adjacent domain search, an individual not jump out of local optimal operation, if the rapid convergence, is likely to fall into local optimum.

Therefore, in order to improve the whale algorithm, add the steps of reconnaissance and search and set the value limit to record The Times when the whale position is not improved. If the quality of the solution is still not improved after limit attempts to change a certain whale position, the solution will be abandoned. The whales use mutation and crossover to break the current local dilemma and scout for new locations. 


\subsection{Termination criterion}

Sets the maximum number of iterations of the whale algorithm. If the current number of iterations reaches the maximum number, the algorithm terminates.

\section{Experimental simulation}

\subsection{Simulation scenes}

The application scheduling scenario is designed as follows: Assume that there are four available satellite resources, and the specific satellite resource information is shown in Table 4. At 00 o 'clock on July 16, 2021, 40 applications for daily tasks are received, and resource scheduling operations need to be performed. Table 5 shows the application requirements of collated tasks. When you set the task start time and required bandwidth, conflicts between tasks are added to reflect the effectiveness of resource scheduling in resolving conflicts between tasks.

Table 4. Satellite communication resources.

\begin{tabular}{cccccccc}
\hline & $\begin{array}{c}\text { Sub-satellite } \\
\text { point }\left({ }^{\circ} \mathbf{E}\right)\end{array}$ & Frequency & $\begin{array}{c}\text { Start frequency } \\
(\mathbf{M H z})\end{array}$ & $\begin{array}{c}\text { End frequency } \\
(\mathbf{M H z})\end{array}$ & $\begin{array}{c}\text { Longitude Latitude } \\
\left({ }^{\circ} \mathbf{N}\right)\end{array}$ & $\begin{array}{c}\text { Half beam } \\
\left({ }^{\circ} \mathbf{E}\right)\end{array}$ & \begin{tabular}{c} 
Angle $\left({ }^{\circ}\right)$ \\
\hline 1
\end{tabular} \\
\multirow{2}{*}{120} & \multirow{2}{*}{$\mathrm{Ku}$} & 12000 & 12020 & 40 & 120 & 5 \\
2 & \multirow{2}{*}{130} & \multirow{2}{*}{$\mathrm{Ku}$} & 12015 & 12040 & 35 & 61 & 5 \\
3 & & 12075 & 12105 & 17 & 90 & 5 \\
4 & & 12147 & 12187 & 15 & 112 & 5 \\
\hline
\end{tabular}

Table 5. Communication task.

\begin{tabular}{|c|c|c|c|c|c|c|c|c|}
\hline & $\begin{array}{c}\text { Bandwidth } \\
\text { (MHz) }\end{array}$ & Priority & Frequency & Start time & End time & $\begin{array}{c}\text { Longitude } \\
\left({ }^{\circ} \mathrm{N}\right)\end{array}$ & $\begin{array}{c}\text { Latitude } \\
\left({ }^{\circ} \mathbf{E}\right)\end{array}$ & $\begin{array}{c}\text { Radial } \\
(\mathrm{Km})\end{array}$ \\
\hline 1 & 2 & 1 & $\mathrm{Ku}$ & $2021 / 7 / 18$ 8:00 & $2021 / 7 / 219: 00$ & 8 & 113 & 1500 \\
\hline 2 & 4 & 3 & $\mathrm{Ku}$ & $2021 / 7 / 1914: 00$ & 2021/7/22 2:00 & 40 & 73.33 & 1000 \\
\hline 3 & 2 & 5 & $\mathrm{Ku}$ & $2021 / 7 / 1812: 00$ & $2021 / 7 / 2312: 00$ & 45 & 80 & 2000 \\
\hline 40 & 4 & 5 & $\mathrm{Ku}$ & $\begin{array}{c}\cdots \\
2021 / 7 / 20 \text { 8:00:00 }\end{array}$ & $\begin{array}{c}2021 / 7 / 30 \\
8: 00: 00\end{array}$ & 31 & 80 & 1500 \\
\hline
\end{tabular}

\subsection{Experimental results and analysis}

WOA and IWOA algorithms were used to solve the resource scheduling problem of communication satellite. The iteration times were set as $=400$, and the population quantity was set as $=100$. In order to eliminate the influence of randomness, the algorithm was run for ten times, with the mean to reflect the algorithm's solving performance and variance to represent the algorithm's stability. The data of ten times of running are shown in Table 6, and Figure 3 is a schematic diagram of the total revenue of ten times of scheduling.

In the scenario, the number of tasks is 40 , and the total revenue sum is 36 . Using WOA algorithm, the average revenue obtained after ten runs is 29.60 , accounting for $82.22 \%$ of the total revenue, and the average execution task is 30 , accounting for $75 \%$ of the total number of tasks. Using IWOA algorithm, the average return obtained from ten runs was 32.83 , accounting for $91.19 \%$ of the total return, and 34 tasks were executed on average, accounting for $85 \%$ of the total number of tasks. IWOA increased the profit value by $8.97 \%$, and the number of tasks increased by 4 on average. In addition, the variance value of the improved algorithm was 0.406 , reduced by $22.22 \%$, which improved the stability of the algorithm. Taking the results of the fifth run as an example, the convergence curves of the 
target values of the two algorithms are shown in Figure 4. It can be seen that IWOA can jump out of local optimum and effectively improve the quality of the solution.

Table 6. Calculation results of scene simulation.

\begin{tabular}{|c|c|c|c|c|c|c|c|c|c|c|c|c|c|}
\hline & & run1 & run2 & run3 & run4 & run5 & run6 & run7 & run8 & run9 & run10 & Average & variance \\
\hline \multirow{2}{*}{ WOA } & target value & $\begin{array}{c}28.7 \\
31\end{array}$ & $\begin{array}{c}28.6 \\
43\end{array}$ & $\begin{array}{c}28.6 \\
88\end{array}$ & $\begin{array}{c}29.5 \\
81\end{array}$ & $\begin{array}{c}29.2 \\
10\end{array}$ & $\begin{array}{c}30.2 \\
87\end{array}$ & $\begin{array}{c}30.1 \\
43\end{array}$ & $\begin{array}{c}30.4 \\
58\end{array}$ & $\begin{array}{c}30.0 \\
21\end{array}$ & 30.201 & 29.60 & 0.522 \\
\hline & \begin{tabular}{|c|}
$\begin{array}{c}\text { Number of } \\
\text { tasks executed }\end{array}$ \\
\end{tabular} & 29 & 29 & 29 & 30 & 29 & 31 & 30 & 31 & 30 & 31 & 29.9 & \\
\hline \multirow{2}{*}{ IWOA } & target value & $\begin{array}{c}33.0 \\
53\end{array}$ & $\begin{array}{c}32.3 \\
44\end{array}$ & $\begin{array}{c}32.0 \\
90\end{array}$ & $\begin{array}{c}33.2 \\
53\end{array}$ & $\begin{array}{c}33.8 \\
60\end{array}$ & $\begin{array}{c}33.3 \\
92\end{array}$ & $\begin{array}{c}32.3 \\
64\end{array}$ & $\begin{array}{c}33.1 \\
49\end{array}$ & \begin{tabular}{|c|}
31.8 \\
59
\end{tabular} & 32.908 & 32.83 & 0.406 \\
\hline & $\begin{array}{c}\text { Number of } \\
\text { tasks executed }\end{array}$ & 34 & 33 & 33 & 35 & 36 & 35 & 33 & 35 & 33 & 34 & 34.1 & \\
\hline
\end{tabular}

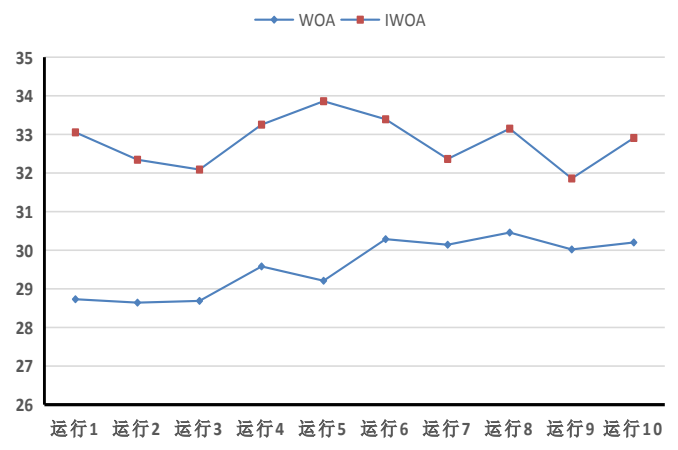

Fig. 3. Schematic diagram of allocation results of communication satellite resource.

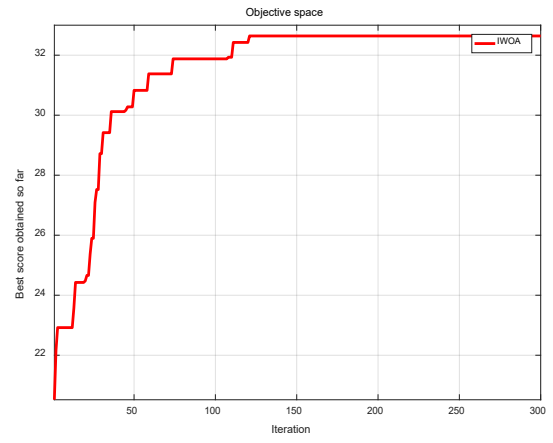

(a) IWOA Running diagram

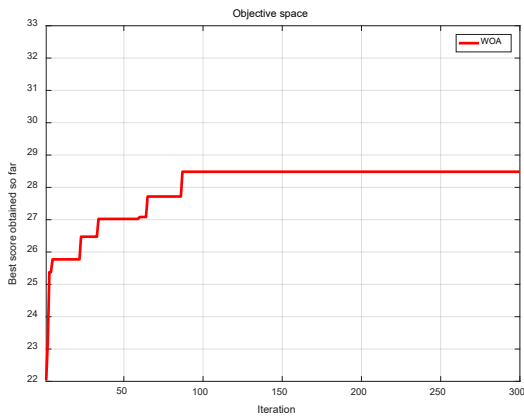

(b) WOA Running diagram

Fig. 4. The 5th algorithm running process diagram.

\section{Conclusion}

In view of the CSRS problem, a communication satellite resource scheduling model is established by analyzing the characteristics of satellite resources and task requirements. and the IWOA algorithm is proposed based on the characteristics of the problem domain, through the reconnaissance and search of individuals trapped in local optimization, the local optimal is broken through and the solution quality is improved. Simulation results show that IWOA algorithm has advantages over WOA algorithm in terms of solution quality and 
stability, which verifies the rationality and effectiveness of the proposed communication satellite resource scheduling model and the improved solution algorithm.

\section{References}

1. Li Honhjun, Liu Rong,Han Fuchun.Research on Task Scheduling Based on Communication Satellite Resources.The 9th China Satellite Communication Broadcasting and Television Technology International Conference and New Equipment Exhibition.2011.

2. He Yan,Zhang Haiyong,Ren Zhong. Research on GEO Satellite Communication Resource-Task Analysis and Modeling Match. Aerospace Control,2014,32(06):44$49+56$

3. Lin Yusheng,Jiang Honglei,Dong Yanlei,Geng Jizhao,Liu Yuexiao.Research of Dynamic Scheduling Method of Communication Satellite Resources Based on Genetic Algorithm.Radio Engineering,2017,47(06):20-23.

4. Mirjalili S, Lewis A. The whale optimization algorithm[J]. Advances in engineering software, 2016, 95: 51-67.

5. Mostafa A, Hassanien A E, Houseni M, et al. Liver segmentation in MRI images based on whale optimization algorithm[J]. Multimedia Tools and Applications, 2017, 76(23): 24931-24954.

6. Prakash D B, Lakshminarayana C. Optimal siting of capacitors in radial distribution network using whale optimization algorithm[J]. Alexandria Engineering Journal, 2017, 56(4): 499-509.

7. Xu Jiya, WANG Yan, JI Zhicheng. Journal of system simulation,2017,29(09):21892197.

8. Wu Kun, Tan Shaochang. Uav route planning based on improved whale optimization algorithm [J]. Acta aeronautica et astronautica sinica,2020,41(S2):107-114.

9. Wang Tao,Ryad Chellali. Whale algorithm with Nonlinear Weights and Convergence factors [J]. Microelectronics and computer,2019,36(01):11-15.

10. Liu Lei, Bai Keqiang, Dan Zhihong, Zhang Song, Liu Zhigui. A whale optimization algorithm for global search strategy [J]. Journal of small microcomputer systems,2020,41(09):1820-1825. 